\title{
PO3-1-53
}

Poster session

\section{Variability of peripheral and central organ activity patterns of mice in an elevated plus maze}

\section{Toya Okonogi', Ryota Nakayama', Takuya Sasaki ${ }^{1,2}$, Yuji Ikegaya $^{1}$}

\author{
${ }^{1}$ Lab. Chem. Pharmacol., Grad. Sch. Pharm. Sci., The Univ. Tokyo, Japan, ${ }^{2}$ Precursory Research for Embryonic \\ Science and Technology, Japan Science and Technology Agency, Japan
}

Elevated plus maze (EPM) tests have been used to assess animal anxiety levels. Little information is known regarding how physiological activity patterns of the brain-body system are altered during EPM tests. Herein, we monitored cortical local field potentials of the prefrontal cortex and hippocampus, electrocardiograms from the intercostal muscle, electromyograms from the dorsal neck muscle, and respiratory signals from the olfactory bulb in individual mice that were repeatedly exposed to EPM tests. Heart rates of the mice were higher in the open arms. In the closed arms, the mice occasionally showed decreased heart and respiratory rates lasting for several seconds or minutes, characterized as lowperipheral activity (LP) states of peripheral signals. The LP states were observed only when the animals were in the closed arms, and the frequencies of the LP states increased as the testing days proceeded. The periods of LP states corresponded with those having significant lower amplitudes of electromyogram. The state more likely occurred in the distal part of the closed arms, which indicates that mice with LP states showed less locomotor activity. During the low-activity states, the delta and theta powers of cortical local field potentials were significantly increased and decreased, respectively. These results demonstrate that cortical oscillations crucially depend on whether an animal exhibits low-activity states in peripheral organs rather than the EPM arm in which the animal is located. These results suggest that combining behavioral tests with physiological makers enables an accurate evaluation of rodent mental states. 